\title{
Satiety induced by bile acids is mediated via vagal afferent pathways
}

\author{
Xiaoyin Wu, Ji-Yao Li, Allen Lee, Yuan-Xu Lu, Shi-Yi Zhou, and Chung Owyang \\ Division of Gastroenterology, Department of Internal Medicine, University of Michigan, Ann Arbor, Michigan, USA.
}

The aim of this study was to elucidate the role and the pathways used by bile acid receptor TGR5 in transmitting satiety signals. We showed TGR5 colocalized with cholecystokinin type A (CCK-A) receptors in a subpopulation of rat nodose ganglia (NG) neurons. Intra-arterial injection of deoxycholic acid (DCA) dose-dependently increased firing rate in NG while a subthreshold dose of DCA and CCK-8 increased firing rates synergistically. TCR5-specific agonist oleanolic acid induced NG neuronal firing in a dose-dependent manner. However, the same units did not respond to GW4064, a nuclear receptor-specific agonist. Quantity of DCA-activated neurons in the hypothalamus was determined by c-Fos expression. Combining DCA and CCK-8 caused a 4-fold increase in c-Fos activation. In the arcuate nucleus, c-Fos-positive neurons coexpressed cocaine and amphetamine regulated transcript and proopiomelanocortin. DCA-induced c-Fos expression was eliminated following truncal vagotomy or silencing of TGR5 in the NG. Feeding studies showed intravenous injection of $1 \mu \mathrm{g} / \mathrm{kg}$ of DCA reduced food intake by $12 \% \pm 3 \%, 24 \% \pm 5 \%$, and $32 \%$ $\pm 6 \%$ in the first 3 hours, respectively. Silencing of TCR5 or CCK-A receptor in the NG enhanced spontaneous feeding by $18 \% \pm 2 \%$ and $13.5 \% \pm 2.4 \%$, respectively. When both TCR5 and CCK-A receptor were silenced, spontaneous feeding was enhanced by $37 \% \pm 4 \%$ in the first 3 hours, suggesting that bile acid may have a physiological role in regulating satiety. Working in concert with CCK, bile acid synergistically enhanced satiety signals to reduce spontaneous feeding.

Authorship note: WX and JYL are co-first authors.

Conflict of interest: The authors have declared that no conflict of interest exists.

Copyright: () 2020, American Society for Clinical Investigation.

Submitted: August 6, 2019

Accepted: June 11, 2020

Published: July 23, 2020.

Reference information: JCI Insight. 2020;5(14):e132400. https://doi.org/10.1172/jici. insight.132400.

\section{Introduction}

TGR5, also known as M-BAR, GPBAR, GPR131, or BG37, is a G protein-coupled receptor containing 7 transmembrane domains that is activated by bile acids. Upon activation, TGR5 transduces signals through the Gs protein and results in production of cAMP $(1,2)$. Widely distributed in the gastrointestinal tract, the liver, the gallbladder, adipocytes, and smooth muscle and immune cells, TGR5 regulates a wide range of physiological functions, including intestinal secretion, gut motility, and glucose homeostasis (3-5). Several lines of evidence show that TGR5 is involved in the regulation of energy metabolism. Watanabe et al. showed that administration of bile acids prevented high fat diet-induced obesity and increased energy expenditure in brown adipose tissue in mice (6). These effects were mediated by TGR5, which activates thyroid hormone-activating enzyme iodothyronine deiodinase type II in brown adipose tissue (BAT). Maruyama et al. observed that female TGR5 ${ }^{-/-}$mice weighed more and had a higher fat content, compared with the WT (7). However, it is not known whether TGR5 is involved in the regulation of food intake.

Bile acids (BA) are secreted into the gastrointestinal tract in the postprandial period, where they facilitate the absorption of lipids and fat-soluble vitamins (8). Primary BA, including cholic acid and chenodeoxycholic acid, are synthesized in the liver. The majority of BA (95\%) are reabsorbed in the terminal ileum and transported back to the liver via the portal vein through a process termed enterohepatic circulation (9). In the terminal ileum and colon, small quantities of primary BA are converted by gut bacteria into secondary BA, of which the most important are deoxycholic acid (DCA) and lithocholic acid (10). In addition to acting as detergents for lipid digestion and absorption, BA are increasingly recognized as metabolic regulators. Our preliminary studies in rats showed that DCA stimulated c-Fos expression in the hypothalamus, raising the possibility that BA may act as a satiety signal.

The gut hormone cholecystokinin (CCK) is synthesized by the small intestinal enteroendocrine I cells. In response to a meal, CCK is released and mediates a number of physiological processes, including pancreatic enzyme secretion, stimulation of gallbladder contraction, and induction of satiety (11). 
CCK also acts as a major neurotransmitter in peripheral nerves as well as the central nervous system. Systemic administration of $\mathrm{CCK}$ at physiological doses resulted in significant increases in satiety, including in human studies (12-14). This action appears to be mediated by CCK type A (CCK-A) receptors on the vagal afferent neurons because pretreatment of rats with a CCK-A receptor antagonist abolished the inhibitory effects of CCK on food intake $(15,16)$. CCK-A receptors are widely distributed in nodose ganglia (NG) and vagal nerve fibers $(17,18)$. However, whether TGR5 is expressed in vagal afferent neurons and colocalized with CCK-A receptor is unknown. Because the vagal NG is a principal site for mediating the satiety actions of a number of gut peptides, we hypothesize that vagal afferent neurons may coexpress both TGR5 and CCK-A receptors and BA may act via the vagus nerve to transmit satiety signals to the hypothalamus. We further propose that BA act synergistically with CCK to enhance the satiety signal following a high-fat meal.

\section{Results}

TGR 5 colocalizes with CCK-A receptor in NG neurons, which are activated by bile acid and $C C K-8$. Double immunofluorescence staining of rat NG with antibodies against the neuronal marker $\mathrm{Hu} \mathrm{C} / \mathrm{D}$ and TGR5 or CCK-A receptor (CCK-AR) showed that $42 \% \pm 4 \%$ of neurons expressed TGR5 while $52 \% \pm 3.8 \%$ of neurons expressed CCK-AR (Figure 1, A and B). Double staining using TGR5 and CCK-AR antibodies demonstrated that $30 \% \pm 2 \%$ of neurons (Hu C/D positive) expressed both TGR5 and CCK-AR (Figure $1, C$ and D). Using reverse transcription PCR (RT-PCR), we showed that TGR5 mRNA was expressed in rat NG (Figure 1E). In addition, we showed that FXR $\alpha$ mRNA was also expressed in NG. However, PRX mRNA was not detectable (Figure 1E), The image of the full, uncut gel is shown in Supplemental Figure 4.

We next investigated whether BA can activate vagal afferent neurons in the NG by performing in vivo single-unit NG recording. We showed a dose-dependent increased firing rate in 18/49 units from 5 rats in response to superior mesenteric artery infusion of DCA $(0.1-10 \mu \mathrm{g} / \mathrm{kg})$ with a threshold dose of $0.5 \mu \mathrm{g} / \mathrm{kg}$ (Figure 2A). Analysis using Spike 2 software demonstrated that $0.5 \mu \mathrm{g}, 1 \mu \mathrm{g}$, and $10 \mu \mathrm{g} / \mathrm{kg}$ DCA triggered a peak firing rate of $6 \pm 0.5,14 \pm 1.2$, and $23 \pm 2$ spikes per 10 seconds, respectively, compared with a basal firing rate of around 1 per 10 seconds (Figure $2 \mathrm{~A}, P<0.05$ for $0.5 \mu \mathrm{g} / \mathrm{kg} ; P<0.01$ for 1 and $10 \mu \mathrm{g} / \mathrm{kg}, n=5$ ).

We then investigated whether there is a synergistic action between CCK and BA on vagal afferent neurons. We first identified those units in the NG that responded to both CCK- 8 and DCA and showed that superior mesenteric artery administration of CCK-8 $(0.069 \mu \mathrm{g} / \mathrm{kg})$ or DCA $(10 \mu \mathrm{g} / \mathrm{kg})$ each triggered neuronal firing in the same unit (Supplemental Figure 1A; supplemental material available online with this article; https://doi.org/10.1172/jci.insight.132400DS1). We next showed that neither a subthreshold dose of DCA $(0.1 \mu \mathrm{g} / \mathrm{kg})$ nor CCK-8 $(0.0057 \mu \mathrm{g} / \mathrm{kg})$ induced significant neuronal firings over baseline. However, when a subthreshold dose of DCA $(0.1 \mu \mathrm{g})$ and CCK-8 $(0.0057 \mu \mathrm{g} / \mathrm{kg}$, superior mesentery artery) was applied, the same units showed a synergistic response, resulting in an increase in firing rates from $1.5 \pm 0.2$ to $26 \pm 3$ spikes $/ 10 \mathrm{~s}(n=5, P<0.01)$ (Figure 2B). Summarized data are shown in Figure 2B, right panel.

To demonstrate that the DCA dose used in our experiment is physiologically relevant, we measured the serum bile acid levels before (basal) and after DCA administration in rats fed a regular chow or after 2 weeks of a high-fat meal (58\% kcal/2 weeks). Superior mesenteric artery injection of $1 \mu \mathrm{g}$ DCA provided a plasma BA level of $38 \pm 2.4 \mu \mathrm{mol} / \mathrm{L}$ (Figure 2C), which was similar to the basal level observed in the high-fat diet (HFD) group $(32.3 \pm 2.1 \mu \mathrm{mol} / \mathrm{L}$, Figure $2 \mathrm{D})$. HFD feeding also elevated serum DCA levels significantly (Figure 2E). DCA administration increased serum DCA levels by 3-fold (Figure 2E). It has been reported that 1.5-2 hours after taking a regular or high-fat meal, the serum total bile acid and DCA levels increase 4- and 6-fold, respectively $(19,20)$. These levels are within the range we observed after intra-arterial infusion of DCA. Therefore, we believe that the DCA doses used in our experiments are physiologically relevant. This is the range similar to that observed after 2 weeks of HFD (Figure 2E).

TGR5 is responsible for bile acid-induced vagal afferent neuron firing. We next sought to determine whether the membrane receptor TGR5 or the nuclear receptor FXR $\alpha$ mediated DCA-induced NG neuronal firing. We showed that superior mesenteric artery administration of oleanolic acid (OA, TGR5-specific agonist) induced NG neuron firing in a dose-dependent manner. However, the same units did not respond to GW4064 (FXR $\alpha$ agonist) at similar doses (Figure 3, A and B). Repeated administration of OA following GW4064 triggered neuronal firing with the same rates, suggesting that the lack of response to GW4064 is not due to synaptic fatigue (Figure 3A). Moreover, silencing TGR5 in the NG abolished DCA-induced increase in neuronal firing (Figure 3B); however, the same units responded to CCK-8 administration. 

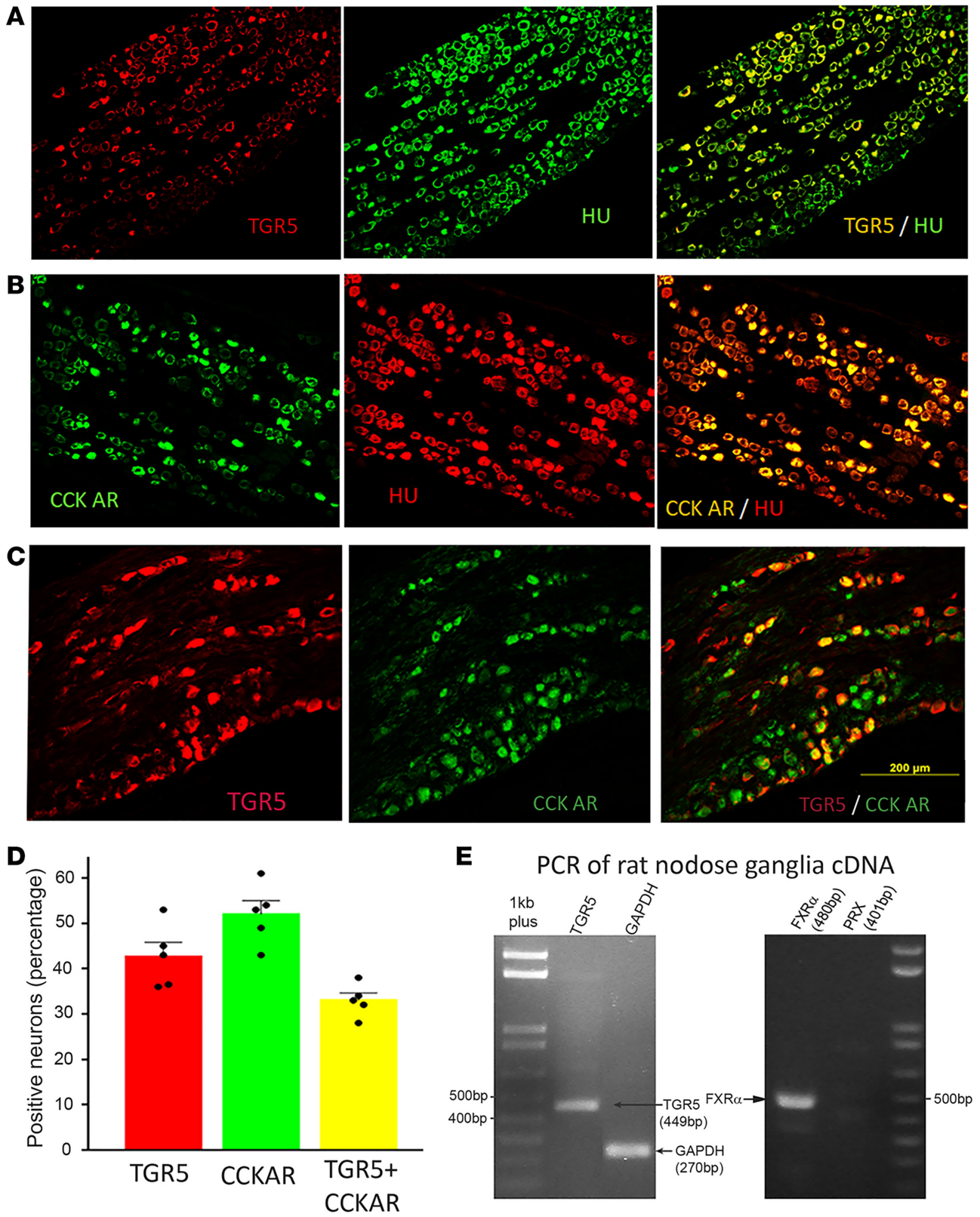

Figure 1. TGR5 and CCK-ARs are coexpressed in rodent NG neurons. (A) Double immunofluorescence staining of TCR5 and neuronal marker Hu C/D as well as merged image. (B) Staining of CCK-AR and Hu C/D as well as merged image. (C) Staining of TCR5 and CCK-AR as well as merged image. (D) Summarized data showing TCR5 was expressed by $42 \%$ $\pm 4 \%$ of neurons, while CCK-ARs were expressed by $52 \% \pm 3.8 \%$ of neurons; both TCR5 and CCK-ARs were expressed by $30 \% \pm 2 \%$ of rodent NG neurons. Three sections per staining were counted, and in total 5 rats were used. (E) TGR5 (left panel) as well as nuclear receptor farnesoid X (FXR $\alpha$, right panel) were expressed in the NG by RT-PCR. However, nuclear receptor pregnane X (PRX) was not detected (right panel).

The results of OA and GW4064 are summarized in Figure 3C. These data indicated that BA act specifically via TGR5 to activate NG neurons.

$D C A$-responsive vagal afferent neurons are cocaine and amphetamine regulated transcript and glutamate immunopositive. To identify the neurochemical phenotype of the NG neurons responding to DCA, neurobiotin-350 was injected into these neurons following recording and triple immunofluorescence was performed. Eight neurons were identified by neurobiotin (Figure 4 shows a representative biotin-positive neuron). The neurobiotin-positive neuron (Figure 4A) expressed both CART (Figure 4, B and C) and glutamate (Figure 4, D and E), as shown in the triple merged image (Figure $4 \mathrm{~F}$ ). We found that all the 8 responsive neurons contained CART and glutamate immunoreactivities, indicating that the DCA-responsive neurons are both CART and glutamate immunopositive. 
A

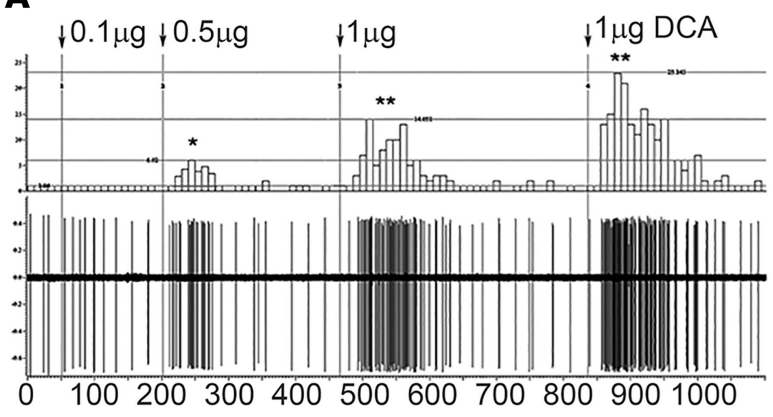

B

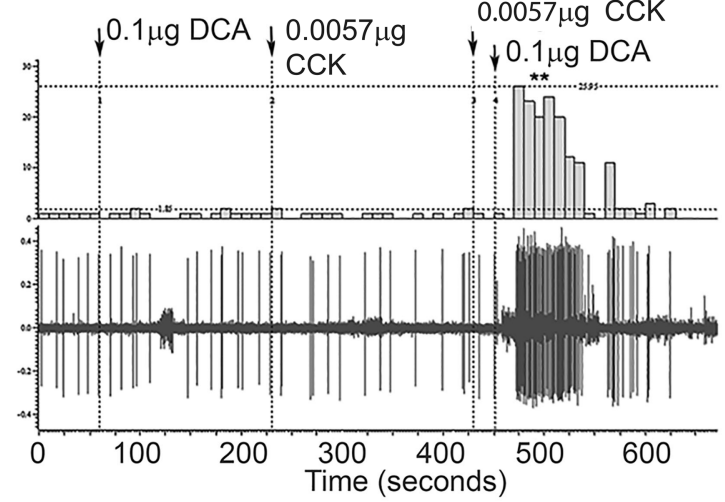

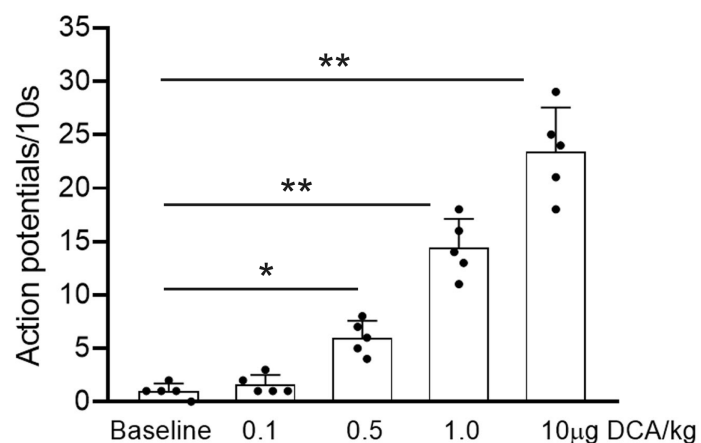

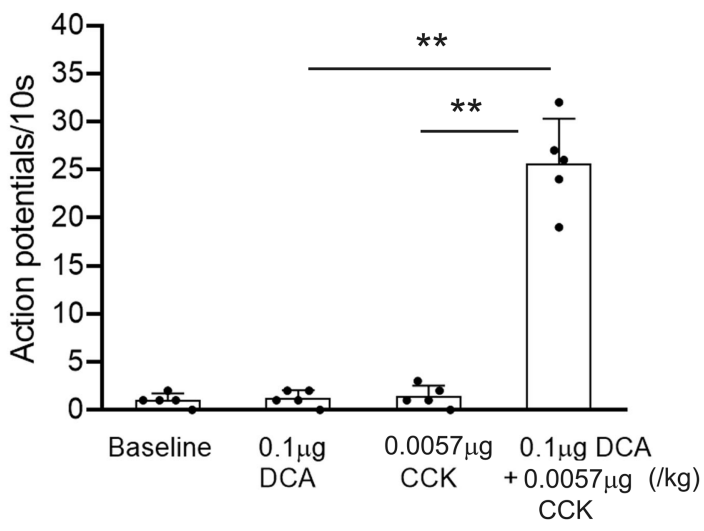

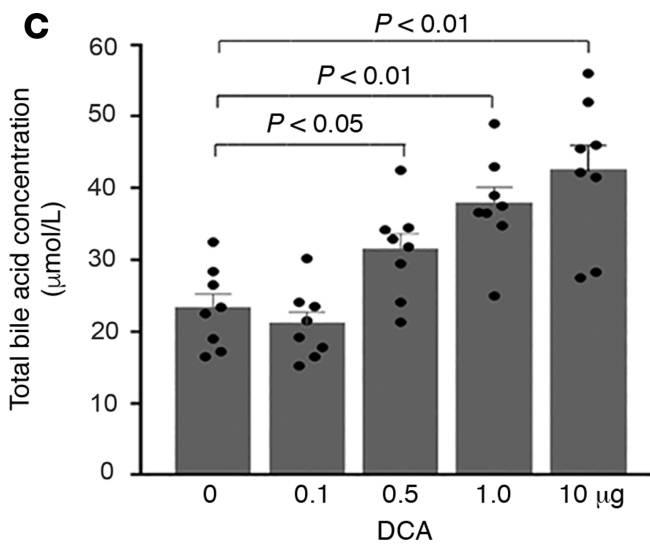

D

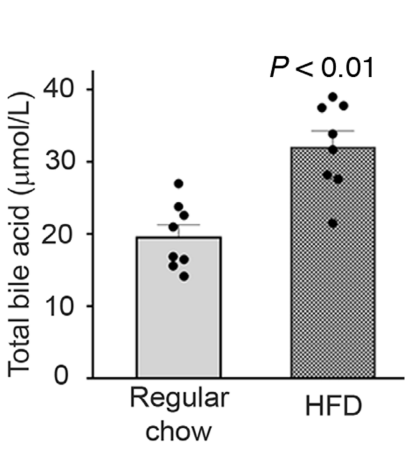

E

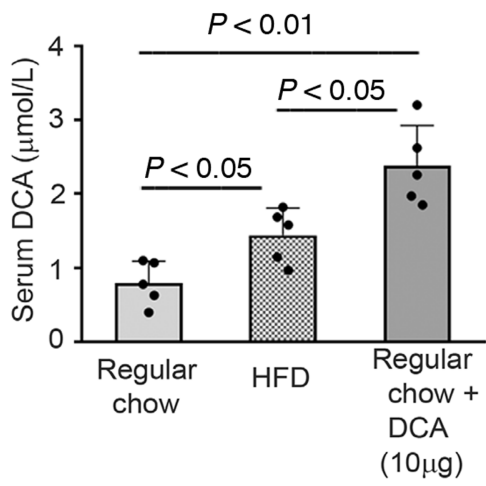

Figure 2. BA and CCK have a synergistic effect on neuronal activation in the NG. (A) Infusion of $0.1 \mu \mathrm{g}, 0.5 \mu \mathrm{g}, 1 \mu \mathrm{g}$, and $10 \mu \mathrm{g} / \mathrm{kg}$ DCA via the superior mesenteric artery showed a dose-dependent increase in the firing rate of vagal afferent neurons in NG. There was a threshold effect seen with a dose of $0.5 \mu \mathrm{g} / \mathrm{kg}$ required for neuronal firing to occur. Right panel shows summarized data. (B) A subthreshold dose of DCA (0.1 $\mu \mathrm{g} / \mathrm{kg})$ combined with CCK-8 $(0.0057 \mu \mathrm{g} / \mathrm{kg})$ resulted in a synergistic effect on vagal afferent neuronal firing compared with either DCA $(0.1 \mu \mathrm{g} / \mathrm{kg})$ alone or CCK-8 $(0.0057 \mu \mathrm{g} / \mathrm{kg})$ alone. Right panel shows summarized data. A and $\mathbf{B}$ show representative recordings of neuronal firing; firing rates per every 10 seconds were determined by analysis using Spike 2 software. ${ }^{*} P<0.05$, ${ }^{*} P<0.01$ compared with basal firing rates. Forty-nine units from 5 rats were recorded. Mann-Whitney $U$ test. (C) Total serum bile acid concentration was measured before and after different doses of DCA injection. One-way ANOVA with Tukey's test. $n=8$. (D) Total serum bile acid concentration was measured in rats fed with either regular chow or high-fat diet (HFD, 58\% kcal fat) after fasting for 6 hours. Unpaired 2-tailed Student's $t$ test. $n=8$. (E) Rats were fasted for 6 hours. Serum DCA concentration was measured in rats fed with regular chow, HFD, or regular chow with intravenous injection of DCA $(10 \mu \mathrm{g} / \mathrm{kg}$, blood was withdrawn 5 minutes after injection). One-way ANOVA with Tukey's test. $n=5$. Doses of DCA and CCK-8 are expressed as micrograms per kilogram.

Neurons in the hypothalamic nuclei involved in the control of appetite are activated by DCA and CCK-8. Because the hypothalamus is a key center for the regulation of appetite, we next examined whether neural signals from the NG initiated by DCA and CCK are relayed to the hypothalamic nuclei involved in the control of appetite. We performed c-Fos staining to investigate the activation of neurons in the hypothalamus. Compared with saline control, administration of DCA $(1 \mu \mathrm{g} / \mathrm{kg}$ iv $)$ induced c-Fos expression in the hypothalamic arcuate nucleus (ARC, Figure 5, A and B), paraventricular nucleus (PVN, Figure 5, E and F), 
A
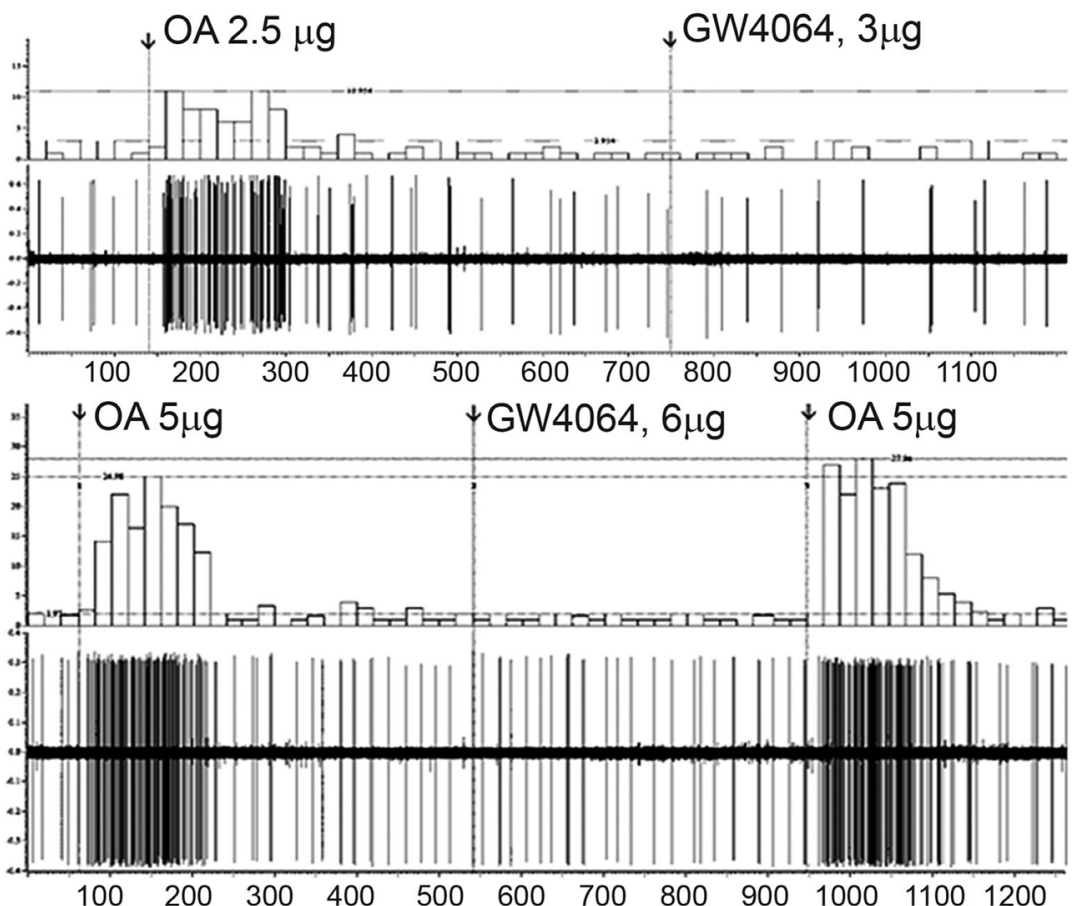

B TGR5 siRNA in NG

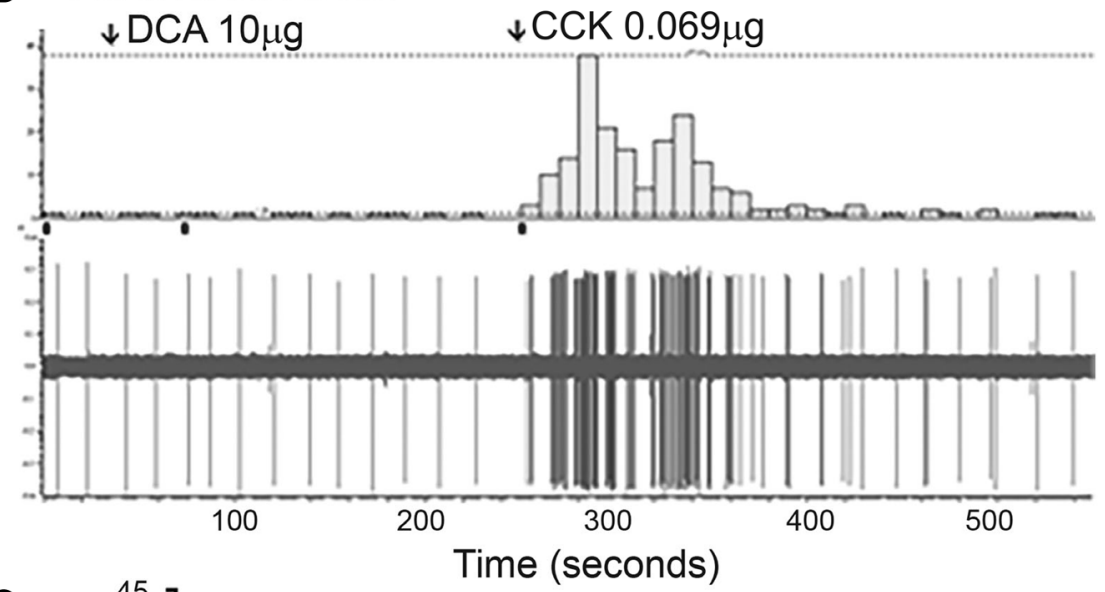

C

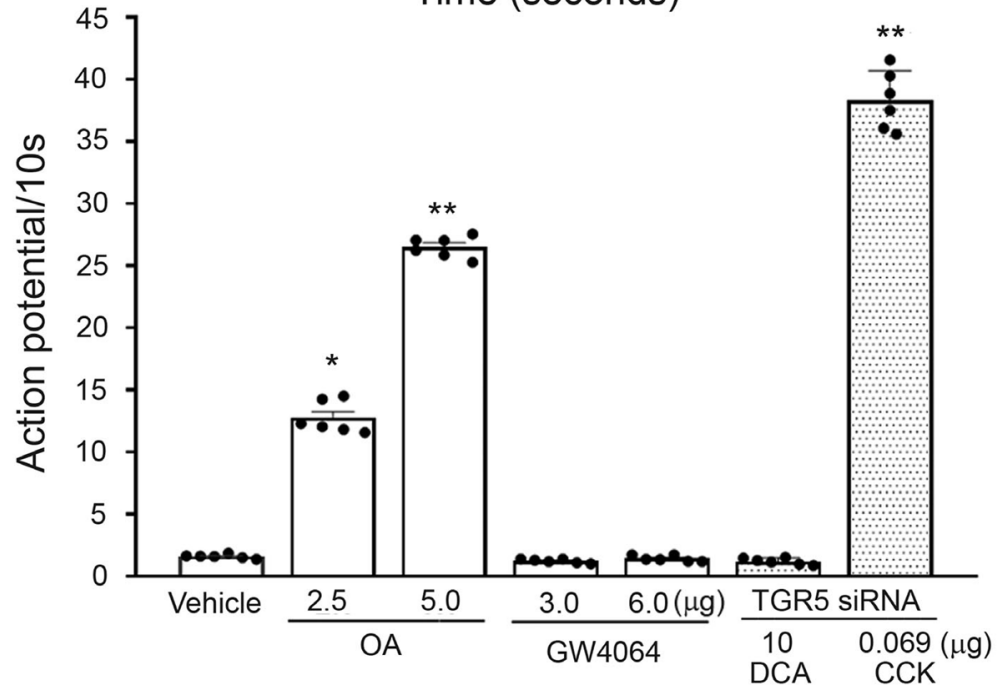

Figure 3. TGR5 is responsible for bile acid-induced vagal afferent neuron firing. (A) A representative recording demonstrates response of vagal afferent neurons to oleanolic acid (OA), a TCR5 agonist, but not to the nuclear receptor agonist CW4064 (top panel), given via the superior mesenteric artery. Vagal afferent neurons responded to the administration of OA following infusion of CW4064 (bottom panel). Thirty-six units from 6 rats were recorded. (B) Silencing of TCR5 in NG abolished the effect of DCA on neuron firing; however, the same unit responded to CCK-8. Twenty-four units from 6 rats were recorded. (C) Summarized data showing administration of $\mathrm{OA}$ resulted in a dose-dependent increase in firing rates per 10 seconds. This effect was not seen with GW4064. Silencing of TCR5 abolished the effect of DCA on neuron firing, but the same units responded to CCK-8. All the doses of drugs are expressed as micrograms per kilogram. ${ }^{*} P<0.05,{ }^{* *} P<0.01$ compared with basal firing, $n=6$. One-way ANOVA with Tukey's test. 

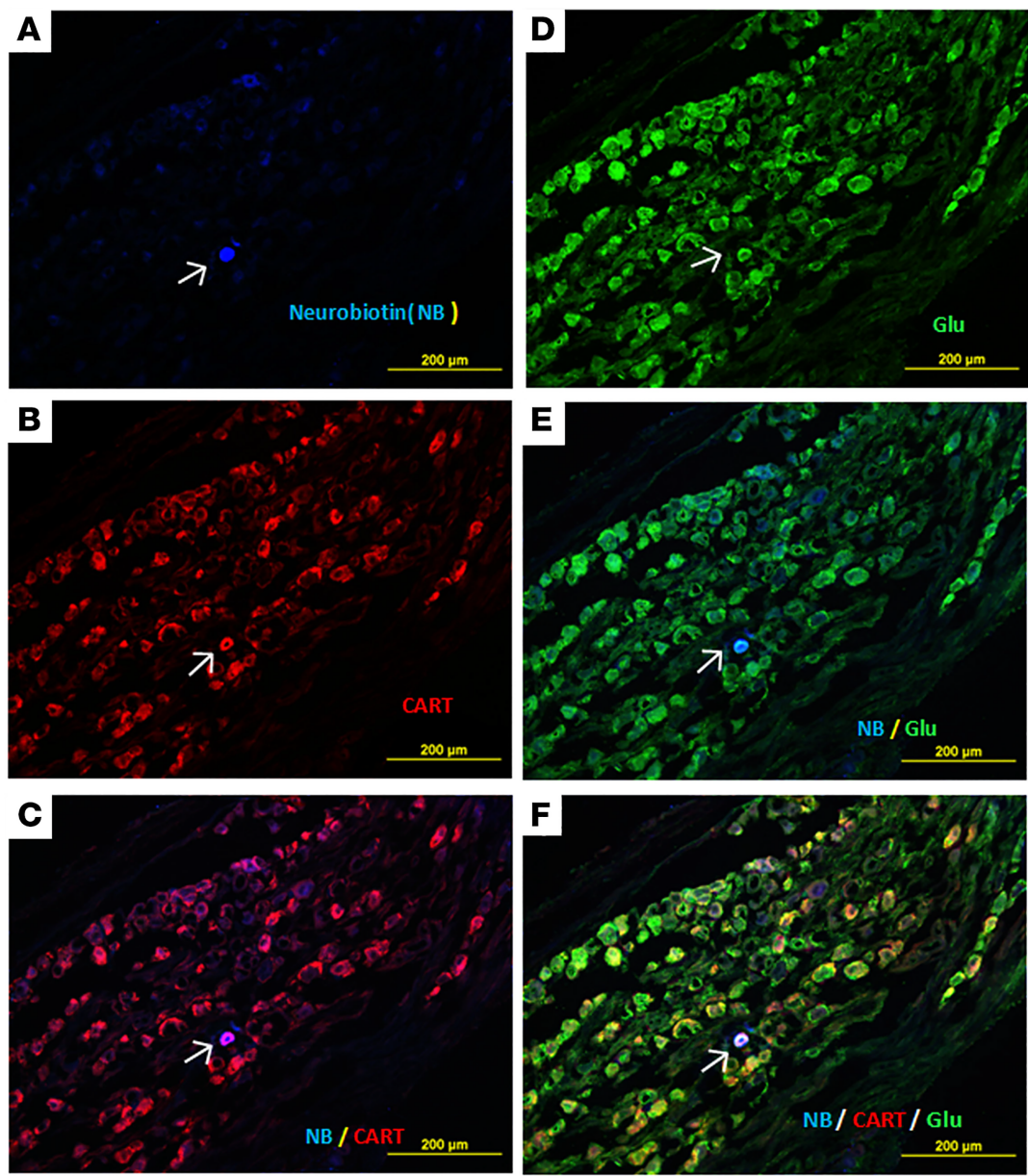

Figure 4. Cocaine and amphetamine regulated transcript and glutamate are expressed by NG neurons. A representative NG neuron is identified after (A) Neurobiotin-350 tracer injection following recording. (B) Cocaine and amphetamine regulated transcript (CART) staining; (C) merged image of neurobiotin and CART immunostaining; (D) glutamate staining; (E) merged image of neurobiotin and glutamate; and (F) triple merged image of neurobiotin, CART, and glutamate staining. Eight neurons from 3 rats were traced and stained.

dorsomedial nucleus (DMD, Figure 5, I and J), and lateral hypothalamus (LH, Figure 5, K and L). The effect of DCA administration on c-Fos expression in the hypothalamic ARC, PVN (Figure 5, C, D, G, and $\mathrm{H})$, DMD, and LH (data not shown) was eliminated following truncal vagotomy or silencing of TGR5 in the NG (Figure 5, C, D, G, and H). Summarized data are shown in Figure 5M. These results indicate that DCA acts via TGR5 in the vagal afferent neurons to induce c-Fos expression in the hypothalamus. Quantitative PCR (qPCR) showed that TGR5 mRNA was knocked down by $65 \% \pm 2.4 \%$ (Supplemental Figure $1 \mathrm{C}$, right). Immunofluorescence staining using TGR5 antibody showed that TGR5 was reduced by $71 \% \pm$ $6 \%$ in the NG with TGR5 siRNA (Supplemental Figure 1, D and E, left).

In line with the synergistic action observed in the NG, low doses of DCA $(1 \mu \mathrm{g} / \mathrm{kg})$ and CCK-8 (3.5 $\mu \mathrm{g}$ ) each evoked a modest increase in c-Fos expression in the ARC and PVN (Figure 6). However, a combination of DCA and CCK-8 at the same doses caused a 3- to 4-fold increase in c-Fos activation in the hypothalamic ARC and PVN (Figure 6), indicating enhanced signaling at the level of the hypothalamus because of synergistic action between CCK-8 and DCA at the NG.

To identify the neurochemical phenotype of neurons activated by DCA in the ARC and PVN of the hypothalamus, we performed double immunofluorescence staining and showed that intravenous administration of DCA $(1 \mu \mathrm{g} / \mathrm{kg})$ activated c-Fos expression in a subgroup of POMC and CART neurons (Figure 7, A-F, arrowheads). POMC and CART neurons are well-recognized satiety-inducing neurons and have been shown to colocalize almost $100 \%$ in the ARC (21-23). In the PVN, administration of DCA $(1 \mu \mathrm{g} / \mathrm{kg})$ stimulated c-Fos expression in a select group of corticotropin releasing factor (CRF) neurons (Figure 7, G-I, arrowheads), 

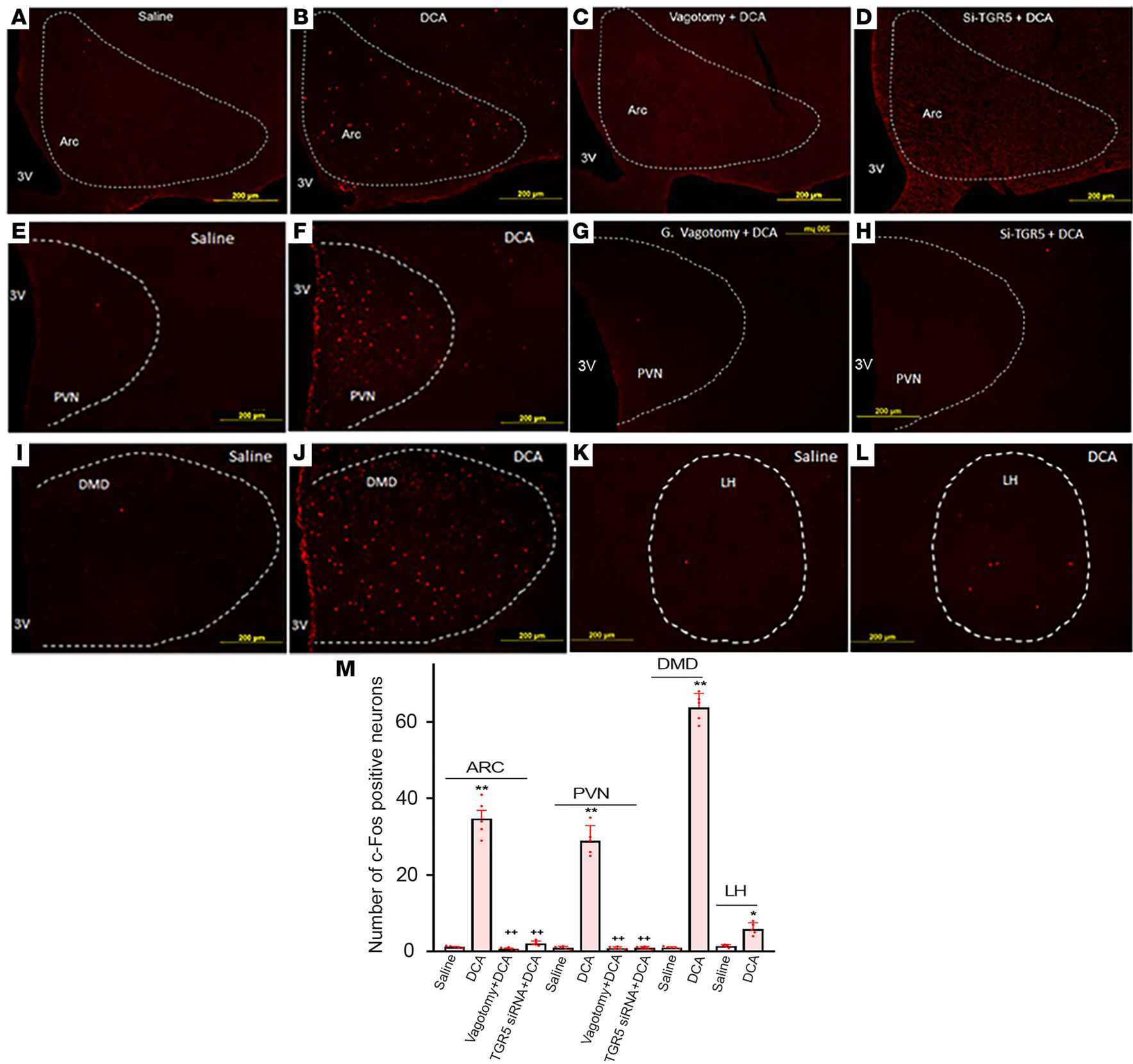

Figure 5. Neurons in the hypothalamus are activated by DCA. Immunofluorescence staining of c-Fos in rat hypothalamus was performed after intravenous injection of saline, injection of DCA $(1 \mu \mathrm{g} / \mathrm{kg})$, or vagotomy + DCA $(1 \mu \mathrm{g} / \mathrm{kg})$ or after silencing of TCR5 (Si-TCR5) in NC + DCA (1 $\mu \mathrm{g} / \mathrm{kg})$. (A-D) ARC. (E-H) PVN. (I and J) DMD. (K and L) LH. ARC, arcuate nucleus; PVN, paraventricular nucleus; DMD, dorsal medial nucleus; LH, lateral hypothalamus; 3V, third ventricle. (M) Summarized data on c-Fos staining. Three sections per rat were counted, and 5 rats were used per group. One-way ANOVA with post hoc Tukey's test was used for statistical analysis. ${ }^{*} P<0.05$, ${ }^{* *} P<0.01$ vs. saline; ${ }^{++} P<0.01$ vs. DCA. $n=5$. One-way ANOVA with Tukey's test.

which are believed to act downstream of POMC/CART neurons (24). We did not observe any colocalization between c-Fos and neuropeptide Y (NPY), an orexigenic neuropeptide in the hypothalamus (Supplemental Figure 2). These data indicate that DCA specifically activates anorexigenic neurons in the hypothalamus.

In the hindbrain, the nucleus tractus solitarius (NTS) is an important relay station for vagal afferent signals. We therefore performed c-Fos staining to investigate the activation of neurons in the NTS. Compared with saline control, intravenous administration of DCA $(1 \mu \mathrm{g} / \mathrm{kg})$ or CCK-8 $(3.5 \mu \mathrm{g} / \mathrm{kg})$ induced a modest increase in c-Fos expression in the NTS. A combination of DCA and CCK- 8 at the same doses caused a much larger increase in c-Fos expression in the NTS. This effect of DCA was abolished after vagotomy or silencing TGR5 in NG (Supplemental Figure 3, A-F). Summarized data on c-Fos staining 

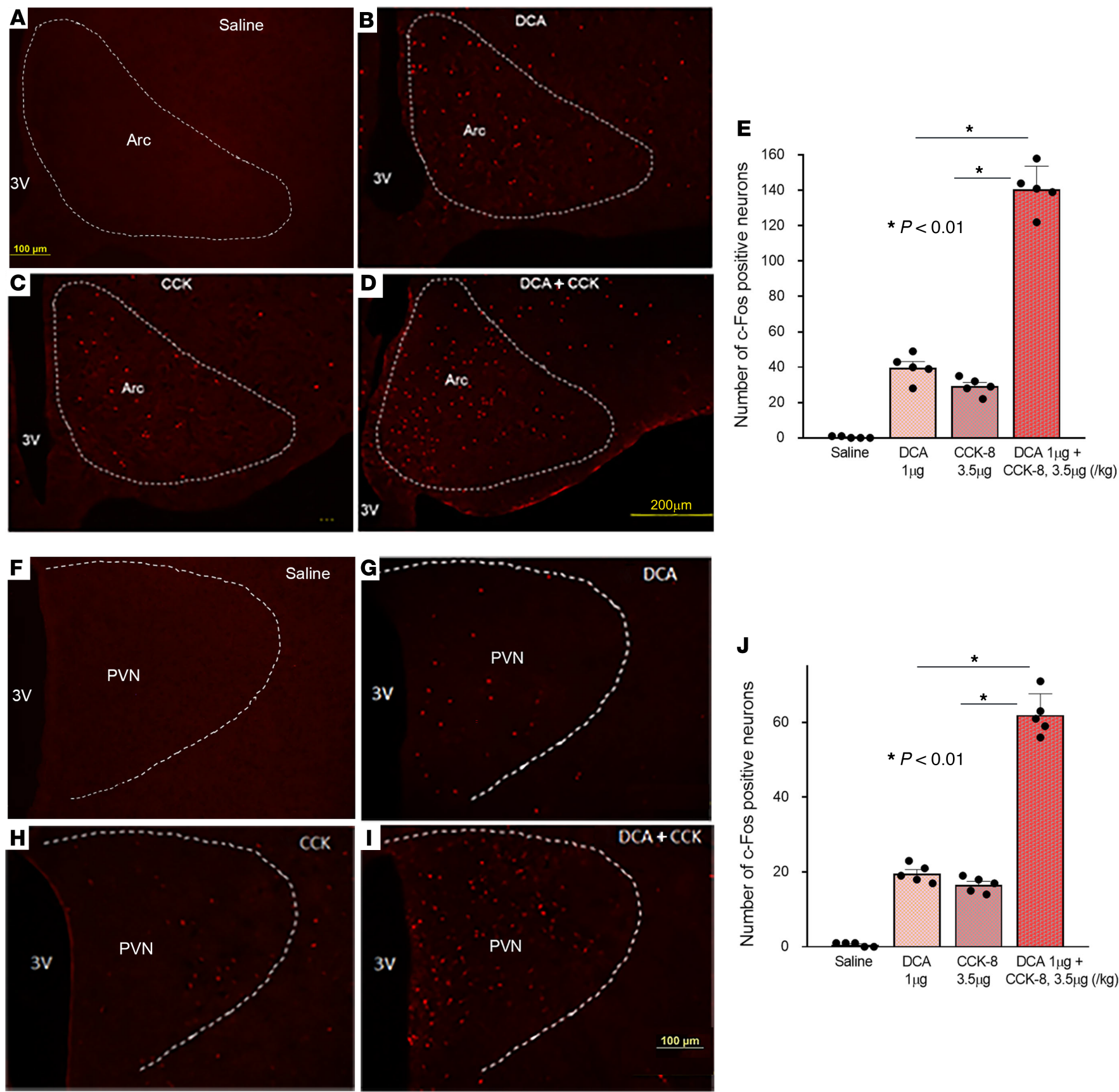

Figure 6. DCA and CCK-8 show a synergistic effect on c-Fos activation in the hypothalamic nuclei. (A-D) c-Fos activation in ARC. (A) Saline control. (B) DCA $(1 \mu \mathrm{g} / \mathrm{kg})$ or (C) CCK-8 $(3.5 \mu \mathrm{g} / \mathrm{kg})$ intravenous injection resulted in a modest increase in c-Fos expression. (D) Injection of both DCA (1 $\mu \mathrm{g} / \mathrm{kg})$ and CCK$8(3.5 \mu \mathrm{g} / \mathrm{kg})$ resulted in a much more robust increase in c-Fos expression. (E) Summary bar graphs showing potentiation effects between DCA and CCK-8 on c-Fos immunoreactivities in the hypothalamic ARC. Three sections per rat were counted, and 5 rats were used per group $(n=5)$. (F-J) Similar results were observed in the PVN. One-way ANOVA with post hoc Tukey's test, ${ }^{*} P<0.05,{ }^{* *} P<0.01 . n=5$.

are shown in Supplemental Figure 3G. These findings indicate that DCA acts via TGR5 in the vagal afferent neurons to induce c-Fos expression in the hindbrain.

DCA inhibits spontaneous food intake via TGR5. We next performed feeding studies to explore the physiological significance of our observations. We showed that intravenous administration of DCA ( $1 \mu \mathrm{g} /$ $\mathrm{kg}$ ) reduced dark phase food intake significantly each hour of the first 3 hours after injection (Figure $8 \mathrm{~A}, P<0.05$ and $0.01, n=8$ ). Silencing of TGR5 in NG increased spontaneous feeding in the first 3 hours in the dark phase by $18 \% \pm 2 \%$ and abolished the DCA-induced $(1 \mu \mathrm{g} / \mathrm{kg})$ inhibition of food intake (Figure 8, B and E), suggesting that TGR5 is responsible for DCA-induced suppression of food intake. 

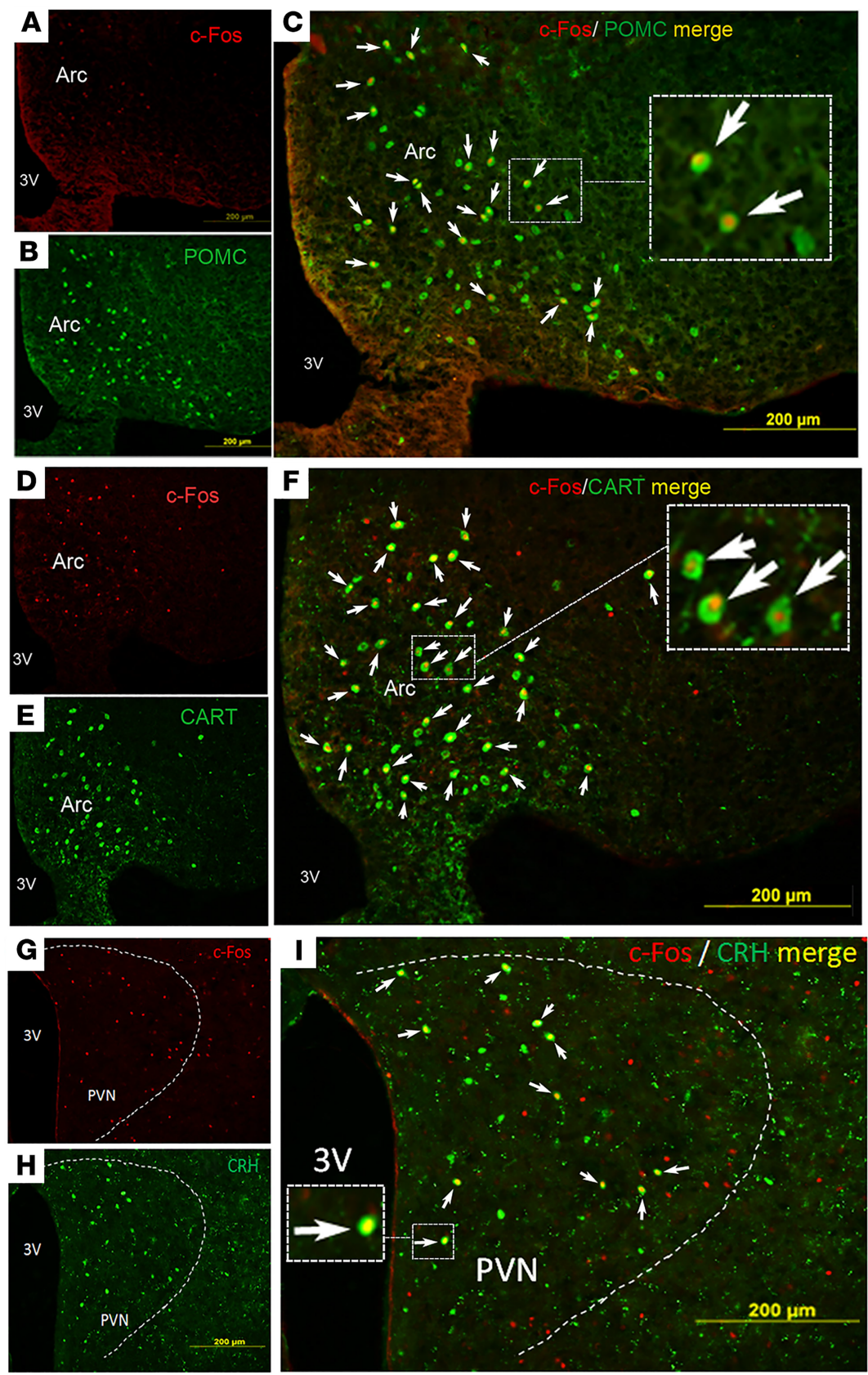

Figure 7. Proopiomelanocortin/CART neurons in ARC and CRF neurons in the PVN are activated by DCA. Double immunofluorescence staining of neurons in the hypothalamic ARC showing (A) c-Fos, (B) proopiomelanocortin (POMC), and (C) merged imaged of c-Fos and POMC staining 1 hour after DCA $(1 \mu \mathrm{g} / \mathrm{kg})$ intravenous injection. Double immunofluorescence staining of neurons in the ARC showing (D) C-Fos, (E) CART, and (F) merged image of c-Fos and CART staining after DCA injection. Double immunofluorescence staining of neurons in the PVN showing (C) c-Fos, (H) CRF, and (I) merged image of c-Fos and CRF staining. Arrows show colocalization of c-Fos with neurons. Insets show that c-Fos is located in the nucleus and neuropeptides in the cytosol of neurons. Three sections per rat were stained and 5 rats were used $(n=5)$.

Silencing of CCK-AR increased spontaneous feeding in the first 3 hours by $13.5 \% \pm 2.4 \%$ (Figure 8B) When both TGR5 and CCK-AR were silenced, spontaneous feeding was enhanced by $37 \% \pm 4 \%$ in the first 3 hours of the dark phase (Figure 8B, $P<0.05$ and 0.01 at $1-3$ hours, respectively, $n=8-13$ ). Silencing of TGR5 or CCK-AR also increased daily food intake by $8 \% \pm 0.2 \%$ and $6 \% \pm 0.2$, respectively, while silencing of both TGR 5 and CCK-AR enhanced daily food intake by $14 \% \pm 0.2 \%$ (Figure $8 \mathrm{C}, P<0.05, n=$ $6-10)$. Moreover, silencing of TGR5 or CCK-AR resulted in additional body weight gains compared with 


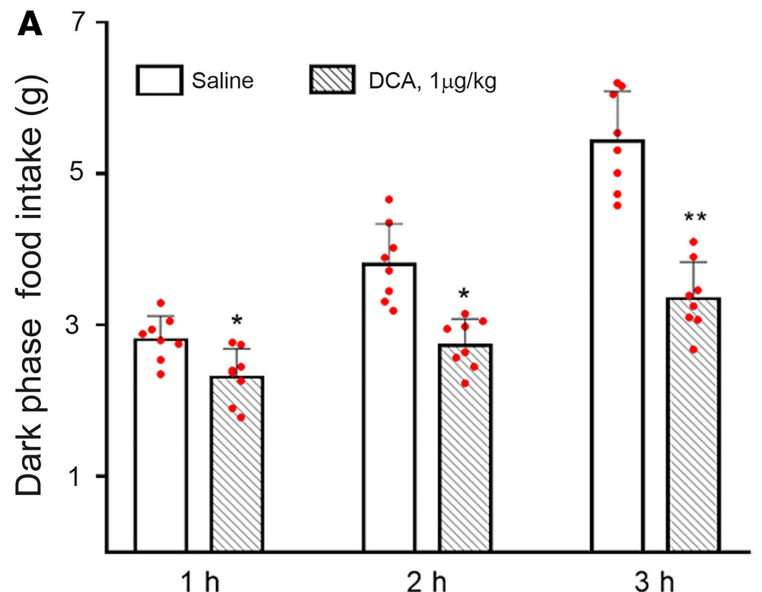

B
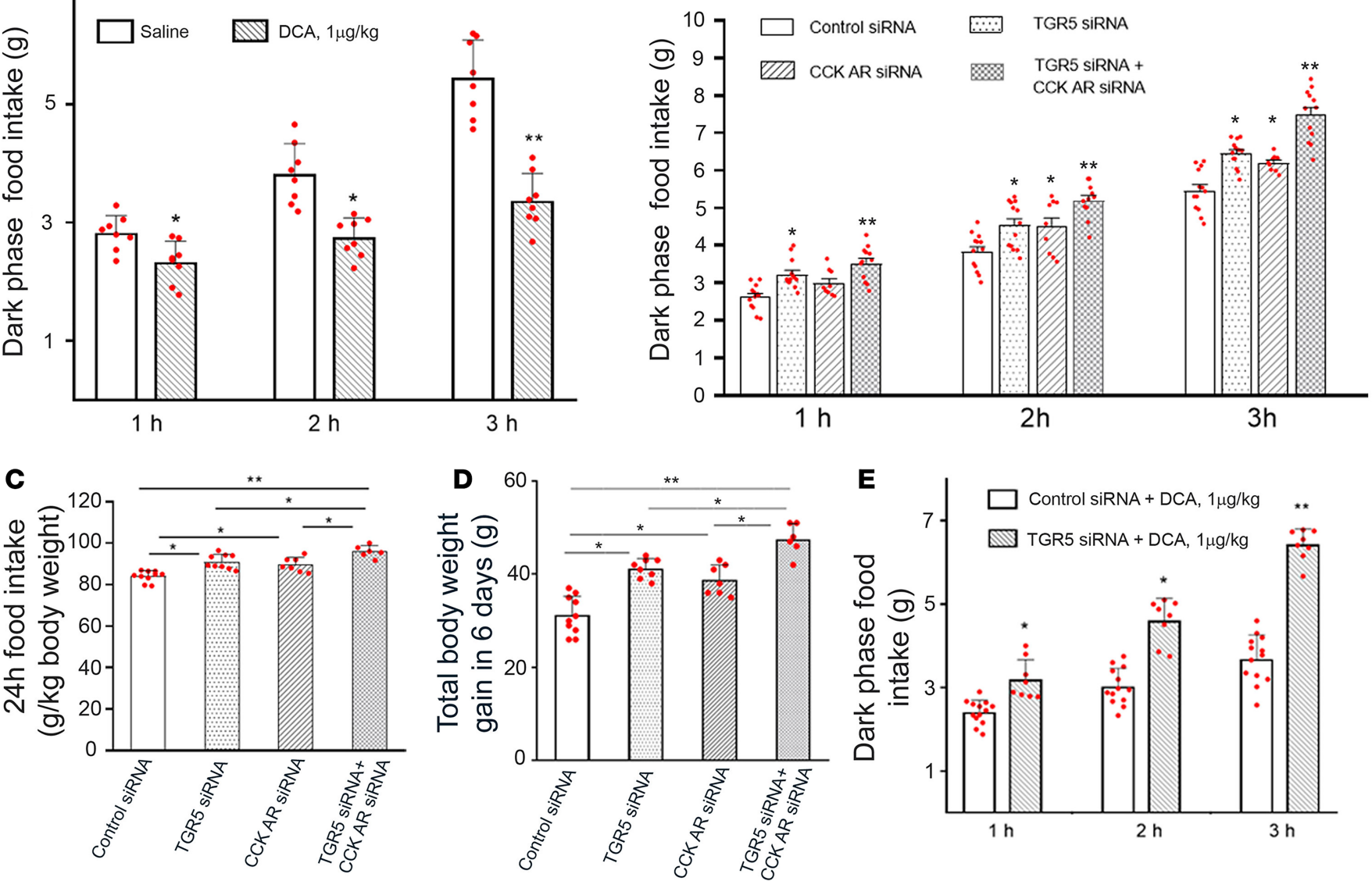

Figure 8. DCA reduces food intake while silencing of TGR5 and CCK-ARs enhances spontaneous feeding. (A) Dark phase spontaneous food intake was measured hourly for 3 hours after intravenous injection of DCA $(1 \mu \mathrm{g} / \mathrm{kg})$. Cumulative food intake significantly decreased at 1,2 , and 3 hours after DCA administration compared with saline (2-way ANOVA with post hoc Tukey's test, ${ }^{*} P<0.05$, ${ }^{*} P<0.01, n=8$ ). (B) One- to three-hour dark phase spontaneous food intake 5 days after knockdown with siRNAs in NG. Silencing of TCR5 increased feeding in the first 3 hours by $18 \% \pm 2 \%$, silencing of CCK-AR increased feeding in the first 3 hours by $13.5 \% \pm 2.4 \%$, while silencing of both TGR5 and CCK-AR enhanced feeding by $37 \% \pm 4 \%$ ( 2 -way ANOVA with post hoc Tukey's test, $\left.{ }^{*} P<0.05,{ }^{*} P<0.01, n=8-13\right)$. (C) Twenty-four-hour food intake 5 days after siRNAs. Silencing of TCR5 or CCK-AR increased daily food intake by $8 \% \pm 0.2 \%$ and $6 \% \pm 0.2 \%$, respectively, while silencing of both TGR5 and CCK AR enhanced daily food intake by $14 \% \pm 0.2 \%$ (1-way ANOVA with post hoc Tukey's test, ${ }^{*} P<0.05,{ }^{*} P<0.01, n=6-10$ ). (D) Total body weight gain (g) 6 days after siRNAs. Silencing of TCR5 or CCK-AR resulted in additional body weight gain compared with control siRNA. Silencing of both TCR5 and CCK-AR enhanced body weight gain further (1-way ANOVA with Tukey's test $\left.{ }^{*} P<0.05, n=6-10\right)$. (E) Compared with control siRNA, silencing of TCR5 abolished DCA-induced ( $1 \mu \mathrm{g} / \mathrm{kg}$, iv) inhibition of feeding, which was measured at day 6 after silencing (2-way ANOVA with post hoc Tukey's test, ${ }^{*} P<0.05,{ }^{* *} P<0.01, n=8-13$ ).

control siRNA. Silencing both TGR5 and CCK-AR enhanced body weight gains even further (Figure 8D, $P$ $<0.05, n=6-10$ ). These data suggest BA released postprandially may have a physiological role to regulate satiety and body weight.

\section{Discussion}

Although well recognized for their role in intestinal micelle formation and lipid digestion, recent studies have indicated that BA may also function as hormone or nutrient signaling molecules to regulate gastrointestinal physiology, glucose homeostasis, and energy metabolism (25). It was demonstrated that TGR5 ${ }^{-1}$ mice gained more weight compared with WT mice (7). However, the exact mechanism responsible for this phenomenon is not clear.

We believe our present study is the first to report that the bile acid receptor TGR5 is present in the NG and colocalizes with CCK-ARs in a subpopulation of vagal sensory neurons. Physiologically, we also demonstrated that BA work in concert with CCK to enhance satiety signals in the hypothalamus and reduce spontaneous feeding in the postprandial period. These actions appear to be mediated by TGR5 on the vagus nerve as silencing of TGR5 in the NG abolished c-Fos activation in the hypothalamic nuclei. 
It is well known that BA may act on membrane TGR5 receptors $(1,2)$ as well as several nuclear hormone receptors, including FXR $\alpha$ and PRX (26-28). These nuclear receptors appear to play important roles in regulating bile acid synthesis, transport, and enterohepatic circulation. On the other hand, TGR5 regulates a wide range of physiological functions, such as glucose and energy metabolism (3-5).

TGR5 expressed on enteroendocrine L cells in the distal small bowel may sense BA, which results in the release of peptide YY (PYY) and glucagon like peptide-1 (GLP-1) (29). Kumar et al. also demonstrated that TGR5 is expressed in the pancreatic $\beta$ cell line MIN6 as well as in mouse and human pancreatic islets (30). Activation of TGR5 expressed in the pancreatic $\beta$ cells resulted in increased insulin release from human islets. Thus, BA may act indirectly to inhibit food intake by stimulating PYY, GLP-1, and insulin release, which can act on the hypothalamic circuits regulating appetite. We showed that vagotomy or silencing TGR5 in the NG abolished DCA-induced neuron activation in the hindbrain and hypothalamus, suggesting TGR5 in vagal afferent neurons plays a key role in conveying satiety signals of BA to the CNS. Our studies provide an alternative mechanism whereby BA may act directly via TGR5 receptors to stimulate CART- and glutamate-containing neurons of the NG, which convey satiety signals to the hypothalamus. We demonstrated that silencing the expression of TGR5 in the NG increased spontaneous feeding and body weight gain, showing the physiological importance of this pathway.

To further delineate the signal transduction mechanisms responsible for the satiety effects of BA, we demonstrated DCA resulted in activation of POMC and CART neurons in the arcuate nucleus. These effects were abolished by vagotomy or silencing the expression of TGR5 in the NG. Both POMC and CART are key neurotransmitters responsible for conveying anorexigenic signals in the hypothalamus and play major roles in the regulation of satiety $(21,22)$. Posttranslational processing of POMC gives rise to $\alpha$-melanocyte stimulating hormone, which acts on melanocortin-3 and -4 receptors in the ARC and PVN to inhibit food intake $(31,32)$. Additionally, the vast majority of POMC neurons in the ARC coexpress CART (23), which is another important anorexigenic peptide. Thus, it is likely that POMC and CART mediate the satiating effects of BA acting via TGR5 on the NG. It is interesting to note that DCA also activates neurons in other hypothalamic nuclei, such as DMD, LH, and PVN. Conceivably, some of these nuclei may be involved in mediating the thermogenic effects of BA on BAT (6).

Secondary BA, such as lithocholic acid and DCA, which are converted by gut bacteria from primary $\mathrm{BA}$ in the terminal ileum and colon, are potent agonists on TGR5 with an $\mathrm{EC}_{50}$ of 0.53 and $1 \mu \mathrm{mol} / \mathrm{L}$, respectively (33). Our studies showed that DCA (10 $\mu \mathrm{g} / \mathrm{kg}$, iv) administration increased serum DCA level to $2.5 \mu \mathrm{mol} / \mathrm{L}$, which is similar to the levels observed after high-fat feeding (Figure $2 \mathrm{E}$ ). These levels are in the range reported after a high-fat meal in humans $(19,20)$, suggesting that the doses of DCA used in our studies are within physiological levels. The vagus nerve innervates the gastrointestinal tract as distal as the midtransverse colon. It is, however, not clear whether TGR5 is present in the terminals of the vagus nerve innervating the intestinal wall. Hence we do not know whether secondary BA can act directly from the lumen. Our study showed that intra-arterial administration of DCA induced firing of vagal afferent neurons, suggesting that these neurons are responding at least to circulating BA.

Postprandially, BA are secreted into the proximal intestine because of stimulation by CCK. Lipids and proteins are major dietary factors that stimulate CCK release (34). Physiological doses of CCK inhibit food intake by reducing meal size (12-14). These actions appear to be mediated by CCK-AR on vagal afferent neurons $(35,36)$. Pretreatment of rats with CCK-AR antagonists abolish the inhibitory effects of CCK on food intake $(15,16,37)$. These anorexigenic signals were previously thought to be due entirely to the actions of CCK on the NG. Our current studies indicate that CCK and BA can each independently inhibit food intake via their actions on the vagal NG. To determine the relative contribution of BA versus CCK for induction of satiety signals, we examined the effect of silencing the expression of CCK-ARs and TGR5 separately with CCK-AR and TGR5 siRNA by electroporation of NG cells. By silencing vagal TGR5 or CCK-AR alone, the first 3 hours' cumulative food intake was increased by $18 \%$ and $13 \%$, respectively. This modest inhibitory action of CCK was likely due to the small increases of circulating CCK levels postprandially. However, concurrent silencing of both CCK-ARs and TGR5 resulted in 37\% increase in 3-hour food intake, suggesting that TGR5 and CCK-AR act synergistically to inhibit short-term food intake. Our c-Fos activation studies showed that DCA and CCK act synergistically to activate neurons in the hypothalamus. However, the interactive effects between BA and CCK appear to be more additive than synergistic on daily food intake and body weight gain. This is not surprising as there are multiple factors other than BA and CCK that participate in the regulation of long-term food intake and body weight. Synergistic interaction 
between BA and CCK on the NG to enhance satiety seems to affect only the immediate postprandial behavior. It is conceivable that in certain conditions, such as bile acid malabsorption, the satiety signals generated by CCK may be weaker without the participation of BA. This may result in hyperphagia to compensate for the energy loss due to fat malabsorption.

In conclusion, we demonstrated for the first time to our knowledge that bile acid receptor TGR5 is present on the vagal NG and approximately one-third of the TGR5 colocalizes with CCK-ARs in the rat NG. DCA and CCK acts synergistically to induce satiety via the POMC/CART hypothalamic neuronal circuit. These satiety effects of BA contribute substantially to the actions of CCK, which results in inhibition of food intake and decreased appetite signals.

\section{Methods}

Materials. All doses are expressed as micrograms/kilogram (DCA) or micrograms/kilogram (CCK-8). DCA and CCK-8 were purchased from MilliporeSigma. Neurobiotin-350 tracer (SP-1151) was obtained from Vector Laboratories. All the primary and secondary antibodies used in this study are listed in Supplemental Table 1.

Animal preparation. Experiments were performed on male Sprague-Dawley rats (220-250 g) (Charles River Laboratories). The rats were housed 3 per cage in a pathogen-free facility and maintained on a 12-hour light/12-hour dark cycle. They were given food and water ad libitum and allowed to acclimate in the facility for 2 days before being randomly assigned to various treatment groups.

Recording of single nodose neuronal activity. Rats were anesthetized with ketamine $(90 \mathrm{mg} / \mathrm{kg}) / \mathrm{xylazine}(10$ $\mathrm{mg} / \mathrm{kg}$ ) and were placed in a small animal stereotaxic frame (Kopf). Body temperature was maintained with a special heating pad. The right nodose ganglion was exposed by a short dorsal approach. Using an operating microscope, the ganglion sheath was removed and separated from the adjacent cervical sympathetic trunk and carotid artery. Protease (type XIV, $0.3 \mathrm{mg} / \mathrm{mL}$ ) was applied to the ganglion for 15 minutes. The recording microelectrodes were pulled from glass capillaries (A-M Systems) using a micropipette puller and microelectrode beveller to obtain tips between 0.08 and $0.1 \mu \mathrm{m}$ in diameter with a resistance of 50-70 M $\Omega$. The beveled glass micropipette filled with $1.0 \mathrm{M} \mathrm{KCl}$ was lowered into the nodose ganglion. Once a nodose ganglion neuron activated by the electrical vagal stimulation was identified, the response of that neuron to superior mesentery artery injection of CCK-8 or DCA was measured (the artery was cannulated before recording with PE10 polyethylene tubing, Becton Dickinson). A reference electrode was placed on a skin incision near the recording electrode. Recordings were taken only from gastrointestinal C-fibers, which were identified according to the following parameters measured in response to electrical stimulation of the abdominal vagus nerve: latency $(60-80 \mathrm{~ms})$, conduction distance between the stimulating electrode and the nodose ganglion $(0.06 \mathrm{~m})$, and conduction velocity $(<1.0 \mathrm{~m} / \mathrm{s})$. Neuronal discharges recoded extracellularly were amplified by an A-M Systems high input-impedance preamplifier, monitored with an oscilloscope and audio monitor, displayed, and stored on an IBM-compatible computer using Axon tape software. The basal discharge was monitored for 2 minutes to confirm the stability of the basal firing frequency. Consistent monitoring of each neuron was ensured by careful study of the firing pattern produced by each neuron and by examining the amplitude and waveform of each spike.

Total bile acid concentration measurement. All groups of rats were food deprived for 6 hours and then sacrificed by $\mathrm{CO}_{2}$ asphyxiation. Fresh serum was collected to measure total bile acid concentration using the Rat Total Bile Acids Assay Kit (Crystal Chem), which is based on enzymatic technology, according to the manufacturer's instructions. Serum DCA was measured at the University of Michigan Metabolomics Core. Briefly, the serum was dried and resuspended for liquid chromatography-mass spectrometry separation by reverse phase chromatography and measured using negative ionization mode triple quadrupole multiple reaction monitoring methods (38).

Intravenous injection, tissue preparation, and immunohistochemistry. For intravenous injection of DCA and CCK-8, the rat external jugular vein was cannulated with PE10 polyethylene tubing under anesthesia with ketamine/xylazine. The tubing was secured to the skin on the back by suture with the tip sealed by fire. The rat was allowed to recover for 7 days before experiments. For intravenous injection, rats were restrained slightly in a restrainer. DCA and CCK- 8 were dissolved in saline and injected intravenously via the cannula at the volume of $0.2-0.3 \mathrm{~mL} /$ rat. Control rats received an equal volume of saline intravenously. Sixty minutes after injection rats were anesthetized with ketamine/xylazine and perfused via the ascending aorta with $200 \mathrm{~mL}$ of phosphate-buffered saline (PBS), followed by $200 \mathrm{~mL}$ of $4 \%$ paraformaldehyde in PBS. 
The bilateral NG and the brain were removed and placed in the same fixative for 2 hours at $22^{\circ} \mathrm{C}$, and then in $25 \%$ sucrose in PBS $(0.1 \mathrm{~mol} / \mathrm{L})$ overnight at $4^{\circ} \mathrm{C}$. The tissue was embedded with $20 \%$ sucrose and Tissue-Tek O.C.T. (2:1, Sakura Finetek). The longitudinal ganglia sections and the coronal brain sections that cover the hypothalamus and the hindbrain were cut measuring $10 \mu \mathrm{m}$ using a precision cryostat (Leica Microsystems). The sections were collected in serially ordered sets, thaw-mounted on Superfrost Plus slides, and stored at $-70^{\circ} \mathrm{C}$. In general, $45-50$ sections were collected.

Double immunofluorescence staining was performed against TGR5, CCK-AR glutamate, and $\mathrm{Hu}$ C/D in NG; against c-Fos, CART, POMC, NPY, and CRF in the hypothalamus; or against c-Fos in the hindbrain, respectively. Briefly, the sections were washed 3 times in wash buffer (PBS containing $0.3 \%$ Triton X-100) and immersed in a blocking solution (5\% normal serum similar to the source of secondary antibody, $1 \%$ BSA, and $0.5 \%$ Triton X-100 in PBS) for 20-30 minutes to inhibit nonspecific binding. The sections were then incubated in a humid chamber for 48 hours at $4{ }^{\circ} \mathrm{C}$ with primary antibodies. After incubation, the sections were washed in PBS buffer and incubated for 1 hour at room temperature with species-specific fluorophore-conjugated secondary antibodies. For negative control, normal IgG instead of primary antibody was used.

Chronic subdiaphragmatic vagotomy. A midline incision was made and the stomach was carefully manipulated to expose the esophagus and gastric cardia. Both the anterior and posterior trunks of the vagus nerve were transected. For the control group, a sham operation was performed where the abdominal vagal nerves were exposed but not cut. Rats were allowed to recover for 7 days after surgery.

$R T-P C R$ and $q P C R$. Rat NG were dissected out and homogenized in TRIzol and total RNA was extracted. Then, $1.5 \mu \mathrm{g}$ RNA was transcribed into cDNA using oligo-dT primers and superscript II (Invitrogen, Thermo Fisher Scientific). All primer pairs span at least 1 intron. RT-PCR primers were rat TGR5: forward primer with EcoRI site (underlined), 5'-GGAATTCCATGGCCCCAAGACCTACAAGAGTG-3', reverse primer with KpnI site, 5'-GGGGTACCCCTGGCAAGCAGGGAGAGGAAACAAA-3'; FXR $\alpha$ : forward, 5'-GGGGCAACTGCGTGATGGAT-3', reverse, 5'-CGACTGCGGACCCTTTGAGC-3'; and PRX forward, 5'-GCGGTCGGCTGGCTTACTGCT-3', reverse, 5'-GCCGTCCGTGCTGCTGAATA-3'. The PCR conditions were $95^{\circ} \mathrm{C}$ for 3 minutes, 1 cycle; $95^{\circ} \mathrm{C}$ for 30 seconds, $57^{\circ} \mathrm{C}$ for 30 seconds, $72^{\circ} \mathrm{C}$ for 1 minute, 36 cycles; $72^{\circ} \mathrm{C}$ for 10 minutes, 1 cycle, with GoTaq DNA polymerase (Promega). The PCR product was visualized in $1 \%$ agarose; the PCR bands were cut out and purified with QIAEX II gel extraction kit (QIAGEN). The purified product was sent for sequencing. qPCR primers for rat TGR5 were forward, 5'-TGCTGTGACTCTTTGATCCTC-3', probe, 5'-/FAM/TGACATCAT/ZEN/GGGTCTTGGCGCA/3IABkFQ/-3', reverse, 5'-CTCTGGGAATGGCTGACAG-3; and CCK-AR: forward, 5'-GCGCCATCTGCAGACCCCTA-3', reverse, 5'-TTGATCACGCCTTCTTGGCTA-3'. qPCR was performed using Bio-Rad CFX-Connect Real Time System with GoTaq DNA polymerase (Promega) for TGR5 and iQ SYBR Green Supermix (Bio-Rad) for CCK-AR. The qPCR conditions were $95^{\circ} \mathrm{C}$ for 3 minutes, 1 cycle, then $95^{\circ} \mathrm{C}$ for 15 seconds, $60^{\circ} \mathrm{C}$ for 1 minute, 40 cycles.

Silencing of TGR5 and CCK-ARs in NG. Thirty minutes before the operation, rats were injected with the analgesic drug carprofen $(5 \mathrm{mg} / \mathrm{kg})$ subcutaneously, then anesthetized with ketamine/xylazine. NG were electroporated with random control siRNA (sc-37007, Santa Cruz Biotechnology), TGR5 siRNA (sc270404), CCK-AR siRNA (sc-108027), or both TGR5 and CCK-AR siRNAs. The in vivo electroporation procedure used in this experiment was previously described (39). Electroporation uses short, high-voltage pulses to overcome the barrier of the cell membrane. Studies have shown that siRNA cannot enter cells without electroporation and that electroporation by itself does not affect the target expression. We have successfully used this technique to transfer siRNA into the NG (40). To measure transfection efficiency, FITC-conjugated control siRNA (sc-36869 Santa Cruz Biotechnology) was used in electroporation. The NG were exposed by a ventral approach. TGR5 siRNA, CCK-AR siRNA, or control siRNA (10 $\mu$ M total, $20 \mathrm{~nL}$ /ganglion) were injected into both left and right NG using a beveled micropipette. After 15 minutes, an isolated pulse stimulator (model 2100, A-M Systems) delivered square wave electric pulses at $50 \mathrm{~V} / \mathrm{cm}$ at $1 \mathrm{~Hz}$ frequency for $20 \mathrm{~ms}$. Transfection and knockdown efficiency were determined by FITC-conjugated control siRNA, RT-PCR, and immunofluorescence staining (Supplemental Figure 1, B-E). Our previous research has shown that siRNA transfection into NG neurons has maximal effect 3 to 6 days postinfection. The optimal conditions for electroporation and GFP expression have been established (40).

Feeding study and body weight measurement. Dark phase (1-3 hours) and 24 hours' spontaneous food intake were measured according to our previous report (41). Briefly, rats were single housed for 3 days for acclimation 
and kept single housed for the duration of the study. To measure spontaneous food intake in the dark phase, preweighed food was provided at 1800 hours, and food intake was monitored every hour for 3 hours. To measure daily food intake, food left was weighed at 1800 hours the following day. To measure body weight gain, body weight was measured before and 6 days after specific mRNA silencing or administration of control siRNA in the NG. To study the effect of intravenous DCA $(1 \mu \mathrm{g} / \mathrm{kg})$ of dark phase 1- to 3-hour food intake, rats were restrained slightly in a restrainer, with DCA, dissolved in saline, or saline $(0.2 \mathrm{~mL})$ injected via the chronic jugular vein cannula 15 minutes before the start of the dark phase (1800 hours).

Statistics. All results are shown as mean \pm SEM for the number of rats indicated. For statistical analyses, unpaired 2-tailed Student's $t$ test, Mann-Whitney $U$ test, and 1-way and 2-way ANOVA with post hoc Tukey's test were used to compare the results from control and experimental groups. A $P$ value less than $0.05(P<0.05)$ was considered significant.

Study approval. All procedures were performed in accordance with NIH guidelines and were approved by the University of Michigan IACUC.

\section{Author contributions}

$\mathrm{CO}, \mathrm{XW}$, and JYL designed the research studies. XW and JYL conducted experiments, acquired and analyzed the data, and wrote the manuscript. YXL and JYL conducted the feeding studies. AL analyzed the data and helped write the manuscript. SYZ conducted experiments. CO designed the experiments and obtained funding.

\section{Acknowledgments}

The studies were supported by NIH grants 2R01 DK058913 and P30 DK34933.

Address correspondence to: Chung Owyang, 3912 Taubman Center, SPC 5362, Department of Internal Medicine, University of Michigan, Ann Arbor, Michigan 48109, USA. Phone: 734.936.4785; Email: cowyang@med.umich.edu.

1. Maruyama T, et al. Identification of membrane-type receptor for bile acids (M-BAR). Biochem Biophys Res Commun. 2002;298(5):714-719.

2. Kawamata Y, et al. A G protein-coupled receptor responsive to bile acids. J Biol Chem. 2003;278(11):9435-9440.

3. Keitel V, Donner M, Winandy S, Kubitz R, Häussinger D. Expression and function of the bile acid receptor TGR5 in Kupffer cells. Biochem Biophys Res Commun. 2008;372(1):78-84.

4. Keitel V, Cupisti K, Ullmer C, Knoefel WT, Kubitz R, Häussinger D. The membrane-bound bile acid receptor TGR5 is localized in the epithelium of human gallbladders. Hepatology. 2009;50(3):861-870.

5. Stepanov V, Stankov K, Mikov M. The bile acid membrane receptor TGR5: a novel pharmacological target in metabolic, inflammatory and neoplastic disorders. J Recept Signal Transduct Res. 2013;33(4):213-223.

6. Watanabe M, et al. Bile acids induce energy expenditure by promoting intracellular thyroid hormone activation. Nature. 2006;439(7075):484-489.

7. Maruyama T, et al. Targeted disruption of G protein-coupled bile acid receptor 1 (Gpbar1/M-Bar) in mice. J Endocrinol. 2006;191(1):197-205.

8. Russell DW. Fifty years of advances in bile acid synthesis and metabolism. J Lipid Res. 2009;50(supp1):S120-S125

9. Houten SM, Auwerx J. The enterohepatic nuclear receptors are major regulators of the enterohepatic circulation of bile salts. Ann Med. 2004;36(7):482-491.

10. Lefebvre P, Cariou B, Lien F, Kuipers F, Staels B. Role of bile acids and bile acid receptors in metabolic regulation. Physiol Rev. 2009;89(1):147-191.

11. Rehfeld JF. Cholecystokinin-from local gut hormone to ubiquitous messenger. Front Endocrinol (Lausanne). $2017 ; 8: 47$.

12. Gibbs J, Young RC, Smith GP. Cholecystokinin decreases food intake in rats. J Comp Physiol Psychol. 1973;84(3):488-495.

13. Lieverse RJ, Jansen JB, Masclee AM, Lamers CB. Satiety effects of cholecystokinin in humans. Gastroenterology. 1994;106(6):1451-1454.

14. Ballinger A, McLoughlin L, Medbak S, Clark M. Cholecystokinin is a satiety hormone in humans at physiological post-prandial plasma concentrations. Clin Sci. 1995;89(4):375-381.

15. Weatherford SC, et al. CCK satiety is differentially mediated by high- and low-affinity CCK receptors in mice and rats. Am J Physiol. 1993;264(2 pt 2):R244-R249.

16. Ebenezer IS. The effects of a peripherally acting cholecystokinin 1 receptor antagonist on food intake in rats: implications for the cholecystokinin-satiety hypothesis. Eur J Pharmacol. 2003;461(2-3):113-118.

17. Grabauskas G, Owyang C. Plasticity of vagal afferent signaling in the gut. Medicina (Kaunas). 2017;53(2):73-84.

18. Burdyga G, et al. Expression of the leptin receptor in rat and human nodose ganglion neurones. Neuroscience. 2002;109(2):339-347.

19. Sonne DP, van Nierop FS, Kulik W, Soeters MR, Vilsbøll T, Knop FK. Postprandial plasma concentrations of individual bile acids and FGF-19 in patients with type 2 diabetes. J Clin Endocrinol Metab. 2016;101(8):3002-3009.

20. Suzuki T, et al. Correlation between postprandial bile acids and body fat mass in healthy normal-weight subjects. Clin Biochem. 
2014;47(12):1128-1131.

21. Mountjoy KG. Pro-opiomelanocortin (POMC) neurones, POMC-derived peptides, melanocortin receptors and obesity: how understanding of this system has changed over the last decade. J Neuroendocrinol. 2015;27(6):406-418

22. Lau J, Herzog H. CART in the regulation of appetite and energy homeostasis. Front Neurosci. 2014;8:313.

23. Vrang N, Larsen PJ, Clausen JT, Kristensen P. Neurochemical characterization of hypothalamic cocaine- amphetamine-regulated transcript neurons. J Neurosci. 1999;19(10):RC5.

24. Schwartz MW, et al. Central nervous control of food intake. Nature. 2000;404(6778):661-671.

25. Hofmann AF, Hagey LR. Bile acids: chemistry, pathochemistry, biology, pathobiology, and therapeutics. Cell Mol Life Sci. 2008;65(16):2461-2483.

26. Makishima M, et al. Identification of a nuclear receptor for bile acids. Science. 1999;284(5418):1362-1365.

27. Parks DJ, et al. Bile acids: natural ligands for an orphan nuclear receptor. Science. 1999;284(5418):1365-1368.

28. Wang H, Chen J, Hollister K, Sowers LC, Forman BM. Endogenous bile acids are ligands for the nuclear receptor FXR/BAR. Mol Cell. 1999;3(5):543-553.

29. Ullmer C, et al. Systemic bile acid sensing by G protein-coupled bile acid receptor 1 (GPBAR1) promotes PYY and GLP-1 release. Br J Pharmacol. 2013;169(3):671-684.

30. Kumar DP, et al. Activation of transmembrane bile acid receptor TGR5 stimulates insulin secretion in pancreatic $\beta$ cells. Biochem Biophys Res Commun. 2012;427(3):600-605.

31. Gantz I, Fong TM. The melanocortin system. Am J Physiol Endocrinol Metab. 2003;284(3):E468-E474.

32. Cone RD. Studies on the physiological functions of the melanocortin system. Endocr Rev. 2006;27(7):736-749.

33. Pols TW, Noriega LG, Nomura M, Auwerx J, Schoonjans K. The bile acid membrane receptor TGR5 as an emerging target in metabolism and inflammation. J Hepatol. 2011;54(6):1263-1272.

34. Green GM, Taguchi S, Friestman J, Chey WY, Liddle RA. Plasma secretin, CCK, and pancreatic secretion in response to dietary fat in the rat. Am J Physiol. 1989;256(6 pt 1):G1016-G1021.

35. Heldsinger A, et al. Cocaine- and amphetamine-regulated transcript is the neurotransmitter regulating the action of cholecystokinin and leptin on short-term satiety in rats. Am J Physiol Gastrointest Liver Physiol. 2012;303(9):G1042-G1051.

36. Zarbin MA, Wamsley JK, Innis RB, Kuhar MJ. Cholecystokinin receptors: presence and axonal flow in the rat vagus nerve. Life Sci. 1981;29(7):697-705

37. Reidelberger RD, Hernandez J, Fritzsch B, Hulce M. Abdominal vagal mediation of the satiety effects of CCK in rats. Am J Physiol Regul Integr Comp Physiol. 2004;286(6):R1005-R1012.

38. Griffiths WJ, Sjövall J. Bile acids: analysis in biological fluids and tissues. J Lipid Res. 2010;51(1):23-41.

39. Saito T. In vivo electroporation in the embryonic mouse central nervous system. Nat Protoc. 2006;1(3):1552-1558.

40. Zhou SY, Lu Y, Song I, Owyang C. Inhibition of gastric motility by hyperglycemia is mediated by nodose ganglia KATP channels. Am J Physiol Gastrointest Liver Physiol. 2011;300(3):G394-G400.

41. Li JY, Wu X, Lee A, Zhou SY, Owyang C. Altered R-spondin 1/CART neurocircuit in the hypothalamus contributes to hyperphagia in diabetes. $J$ Neurophysiol. 2019;121(3):928-939. 